\title{
Tumor-enhancing activity of Basigin expression in gallbladder carcinoma and its prognostic role
}

\author{
GUANG-FA XIAO and HUI-HUAN TANG \\ Department of General Surgery, Xiangya Hospital, Central South University, Changsha 410008, P.R. China
}

Received December 23, 2008; Accepted March 3, 2009

DOI: $10.3892 / \mathrm{mmr} 00000128$

\begin{abstract}
Basigin (EMMPRIN/CD147) is a multifunctional membrane glycoprotein that is overexpressed in many solid tumors and is involved in tumor invasion and angiogenesis. The main purpose of this study was to investigate the tumorenhancing activity of Basigin in a gallbladder carcinoma (GC) cell line and in primary GC tissues. A system that blocks Basigin in the human primary GC cell line GBC-SD was developed using RNA interference. GBC-SD cells were transfected with the small interfering RNA that target Basigin, then the proliferative, invasive and migration activities of the cells were assayed in vitro. Additionally, tissue samples from 98 patients with GC and 26 patients with chronic cholecystitis were stained with anti-Basigin antibody for immunohistochemical analysis. Furthermore, the association of Basigin expression with the clinicopathological characteristics and prognosis of the patients was analyzed. siRNA-treated GBC-SD cells exhibited significantly decreased growth ability, invasion and migration capacities compared to control cells in vitro. Moreover, clinicopathological analysis demonstrated that the intensity of Basigin staining in cancerous tissue was significantly associated with the histological type $(\mathrm{p}=0.02)$, distant metastasis $(\mathrm{p}<0.01)$ and Nevin stage $(\mathrm{p}<0.01)$ of GC. A proportional hazards model revealed the survival rate of patients with stronger Basigin expression to be the lowest $(\mathrm{p}<0.01)$. These results suggest that Basigin is a prognostic marker and potential therapeutic target for patients with GC.
\end{abstract}

\section{Introduction}

Gallbladder carcinoma (GC) is the most common malignancy of the biliary tract. Metaplastic changes have recently attracted great attention as potential key factors underlying the mechanism of GC carcinogenesis. Although radiological and surgical techniques have been rapidly developed, the longterm survival of GC patients is poor. Compared to other

Correspondence to: Dr Hui-Huan Tang, Department of General Surgery, Xiangya Hospital, Central South University, Changsha 410008, P.R. China

E-mail: csxgf2008@126.com

Key words: gallbladder carcinoma, Basigin, RNA interference, immunohistochemistry, clinicopathology, prognosis common types of cancer, the identification of GC prognostic markers has not been extensively pursued, as this disease has a relatively low incidence and is usually diagnosed during its later stages $(1,2)$. Molecular markers, such as p53, MUC1 and MGMT, have been demonstrated to be related to GC progression (3-5). However, most have only been identified in single studies comprising a small number GC cases, and thus have limited value in GC diagnosis. Consequently, the identification of reliable molecular markers may provide important prognostic information and facilitate the identification of adequate therapeutic targets.

Basigin, also known as extracelluar matrix metalloproteinase inducer (EMMPRIN/CD147), is a highly glycosylated member of the immunoglobulin superfamily that is expressed on the surface of many malignant tumor cells and functions as an upstream modulator of matrix metalloproteinase (MMP) production in the local tumor environment (6-8). Recently, it was demonstrated that Basigin may bind and regulate several tumor-related signalling proteins and stimulate cell proliferation and survival (9). Here, we show that the overexpression of Basigin in a GC cell line enhanced cell growth, invasion and migration capacities. We also demonstrate that Basigin is expressed in a heterogeneous pattern in GC tissues compared with chronic cholecystitis tissues. Furthermore, we show that the analysis of Basigin expression can be utilized as a prognostic indicator for GC, independent of several commonly used markers.

\section{Materials and methods}

Cell culture. The human GC cell line GBC-SD and the normal human epithelial cell line HGBEC were obtained from the National Cell Bank of the Cell Institute of Shanghai, China, and were cultured in 90\% DMEM (Gibco) supplemented with antibiotics (1x penicillin/streptomycin $100 \mathrm{U} / \mathrm{ml}$, Gibco) and $10 \%$ FBS (Gibco). Cells were incubated in a humidified atmosphere containing $5 \% \mathrm{CO}_{2}$ at $37^{\circ} \mathrm{C}$.

Patient and tissue samples. A total of 98 fresh GC tissues were obtained from 58 male and 40 female patients (aged 36-78 years, mean \pm SD 52.66 \pm 11.06 years; Nevin stages I-V) immediately after cholecystectomy and were paired with 26 chronic cholecystitis tissues obtained from Xiangya Hospital of Central South University of Changsha, China. None of the patients recruited had undergone chemotherapy or radiotherapy prior to the surgery. Samples obtained from carcinoma tissue 
were fixed in $10 \%$ formalin and routinely processed for paraffin embedding. Histological sections (4- $\mu \mathrm{m}$ thick) were stained with hematoxylin and eosin, as well as immunoperoxidase procedures. The histological sections were independently reclassified by two experienced pathologists according to histological type and Nevin staging. The 98 patients were subject to a 5-year follow-up. Patients that succumbed to diseases other than GC or to unexpected events were excluded from the sample.

The study was approved by the Research Ethics Committee of Xiangya Hospital of Central South University of Changsha, China. Informed consent was obtained from the patients. The specimens were handled in a blinded manner according to ethical and legal standards.

Construction of small interfering RNA targeting Basigin. Based on the Basigin cDNA sequence in GenBank, we used BLAST to design a pair of nucleotide oligos containing endonuclease restriction sites at both ends. Synthesis was performed by Shanghai Pharmnet Biotechnologies (Shanghai, China). Oligos were annealed and ligated with linearized pSUPER using T4DNA ligase. The recombinant plasmid was named pSUPER/Basigin small interfering RNA (siRNA) and was sequenced and identified by PCR and restriction endonuclease digestion.

Transfection of siRNA. The transfection of siRNA was performed to silence Basigin expression. GBC-SD cells were incubated in appropriate antibiotic-free medium with $10 \%$ fetal bovine serum (Gibco), then transferred to a 6-well tissue culture and incubated at $37^{\circ} \mathrm{C}$ in a $\mathrm{CO}_{2}$ incubator to obtain $60-80 \%$ confluence. The cell cultures were transfected with recombinant plasmid $\mathrm{pSUPER/Basigin} \mathrm{siRNA}$ or empty vector in serum-free medium using Lipofectamine 2000 (Invitrogen, Carlsbad, CA), prepared according to the manufacturer's protocol. Cells were collected $72 \mathrm{~h}$ after transfection. Specific silencing of the targeted gene was confirmed by reverse transcription-polymerase chain reaction (RT-PCR) analysis.

Semiquantitative RT-PCR analysis. For the semiquantitative RT-PCR analysis of Basigin mRNA levels, total RNA was isolated from the GBC-SD and HGBEC cells using TRIzol (Invitrogen), and cDNA was synthesized using an RT-PCR kit (Takara, Japan) according to the manufacturer's instructions. The primers sequences were as follows: Basigin, F: 5'-GAG AGCTTGCGAAACTGGTC-3', R: 5'-AACCAACACAGG ACCCAG-3' (492 bp); ß-actin, F: 5'-ACTGGAACGGTG AAGGTGACAG-3', R: 5'-GGTGGCTTTTAGGATGGC AAG-3' (161 bp). PCR analysis was performed under the following conditions: denaturation at $94^{\circ} \mathrm{C}$ for $1 \mathrm{~min}$, then 30 cycles of denaturation for $20 \mathrm{sec}$ at $97^{\circ} \mathrm{C}$, annealing for $20 \mathrm{sec}$ at $64^{\circ} \mathrm{C}$, and extension for $20 \mathrm{sec}$ at $72^{\circ} \mathrm{C}$. The amplified products were analyzed by agarose gel electrophoresis on a $1.0 \%$ gel, followed by ethidium bromide staining. PCR products $(1 \mu \mathrm{l})$ were electrophoresed on $1.5 \%$ agarose gels, and the gray scale ratio of Basigin/ $/$-actin was calculated.

MTT assay. GBC-SD, control RNAi and Basigin/RNAi cells in $200 \mu \mathrm{l}$ DMEM were seeded in duplicate into each well of 96-well culture plates. After 24, 48, 72 and $120 \mathrm{~h}$ of culture,
$100 \mu 1$ of 3-(4,5-dimethylthiazol-2-yl)-2,5-diphenyltetrazolium bromide (MTT, $5 \mathrm{mg} / \mathrm{ml}$, Sigma) solution $(5 \mathrm{mg} / \mathrm{ml}$ in PBS) was added. After a 4 -h incubation at $37^{\circ} \mathrm{C}$ in $5 \% \mathrm{CO}_{2}$, $100 \mu 1$ DMSO (Gibco) was added to each well. The dark blue crystals of MTT-formazan were dissolved by shaking the plates at room temperature for $10 \mathrm{~min}$, then absorbance was measured on a Bio-Rad Microplate Reader (Bio-Rad, Hercules, CA) using a test wavelength of $490 \mathrm{~nm}$ and a reference wavelength of $630 \mathrm{~nm}$. Each experiment was performed in triplicate. Growth rate $(\%)=$ A490 (transfectants)/A490 (RNAi control) $\times 100 \%$.

Invasion and migration assays. GBC-SD, control RNAi and Basigin/RNAi cells were labeled with the fluorescent lipophilic tracer DiI (Molecular Probes, Eugene, OR) and seeded at a density of 50,000 per $6.4-\mathrm{mm}$ diameter well in BD Falcon Individual HTS Fluoroblok Cell Culture inserts in 24-well companion plates (BD Biosciences, San Jose, CA). The percentage of cells moving through the $8 \mu \mathrm{mol} / \mathrm{l}$ pore Fluoroblok membrane was estimated by measuring the fluorescence generated from below at various time points after seeding. For invasion measurements, the membrane was coated with $100 \mu \mathrm{g}$ Matrigel in a 30- $\mu 1$ volume per well. Experiments were carried out in triplicate on three separate occasions.

Immunohistochemical staining and assessment. Immunohistochemical staining was carried out using the avidin-biotin method and a commercially available kit (Vectastain Elite $\mathrm{ABC}$ kit, Vector Laboratories, Burlingame, CA). One paraffinembedded block of tissues was selected from each patient and cut into $4-\mu \mathrm{m}$ sections. The sections were deparaffinized and treated with methanol containing 3\% hydrogen peroxide for $10 \mathrm{~min}$, then antigen retrieval was conducted in a microwave oven at $95^{\circ} \mathrm{C}$ for $5 \mathrm{~min}$ with cooling at $25^{\circ} \mathrm{C}$ for $2 \mathrm{~h}$. After washing in PBS, blocking serum was applied for $10 \mathrm{~min}$. The sections were incubated with anti-Basigin monoclonal antibody (Santa Cruz Biotechnology, CA) overnight at $4^{\circ} \mathrm{C}$. After washing in PBS, a biotin-marked secondary antibody was applied for $10 \mathrm{~min}$ followed by a peroxidase-marked streptavidin for an additional $10 \mathrm{~min}$. The reaction was visualized using 3,3'-diaminobenzidine tetrahydrochloride. The nuclei were counterstained with hematoxylin. Positive and negative immunohistochemistry controls were routinely used. Reproducibility of staining was confirmed by reimmunostaining via the same method in multiple randomly selected specimens.

To evaluate the expression of Basigin, three independent observers without knowledge of the clinicopathologic data examined the immunostained slides. The number of Basiginpositive cells that showed immunoreactivity on the cell membranes and cytoplasm in 10 representative microscopic fields were counted, and the percentage of positive cells was calculated. The criteria used for assessment were as previously reported (10). In brief, expression was classified as 0 (negative), $<5 \%$ of tumor cells stained; 1 (low), $5-75 \%$ of tumor cells stained; and 2 (high), $>75 \%$ of tumor cells stained.

Statistical analysis. SPSS13.0 software for Windows (SPSS Inc., USA) and SAS 9.1 (SAS Institute, NC) were used for 

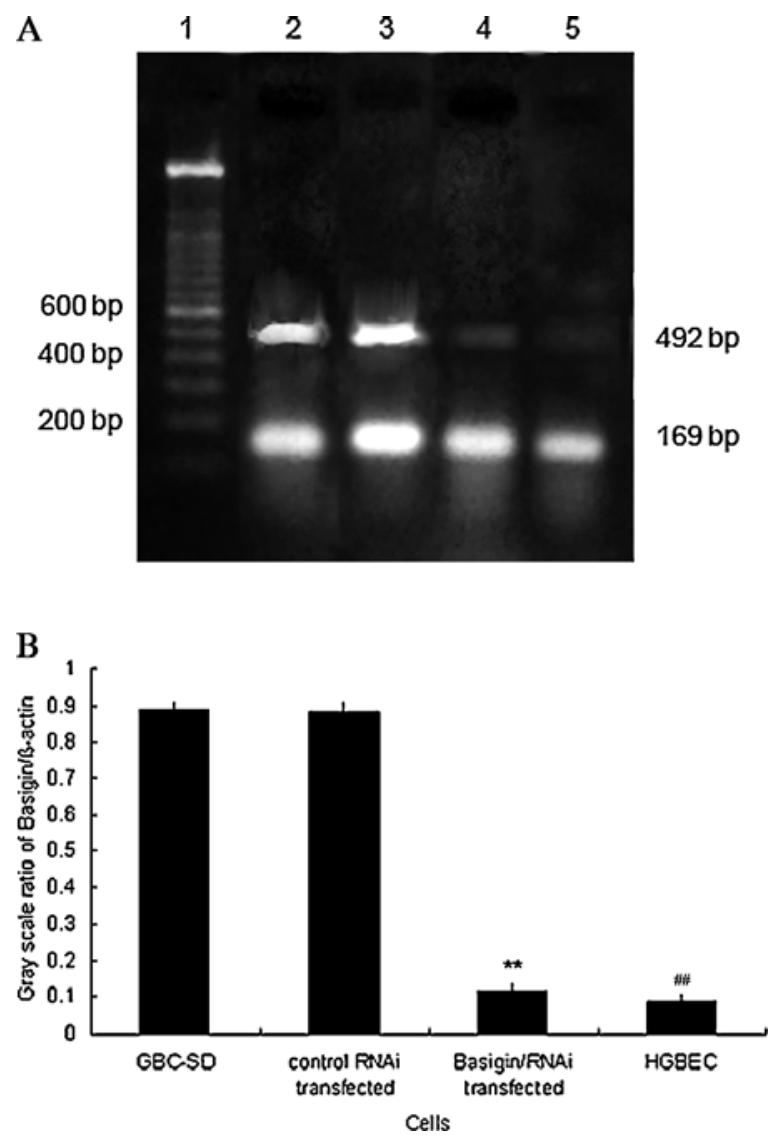

Figure 1. Semiquantitative RT-PCR of Basigin. (A) RT-PCR products of total RNA in GBC-SD, control RNAi, Basigin/RNAi and HGBEC cells were electrophoresed on 1.5\% agarose gels as described in Materials and methods (Basigin, $462 \mathrm{bp}$; ß-actin, $169 \mathrm{bp}$ ). Lane 1, marker; lane 2, GBC-SD cells; lane 3, control RNAi transfected GBC-SD cells; lane 4, Basigin/RNAi transfected GBC-SD cells; lane 5, HGBEC cells. (B) Comparison of gray scale ratio of Basigin/ $/$-actin in GBC-SD, control RNAi, Basigin/RNAi and HGBEC cells. ${ }^{* *}<<0.001$ compared to untransfected GBC-SD cells and control RNAi transfected GBC-SD cells; \#\# $<0.001$ compared to GBC-SD cells.

statistical analysis. Continuous variables were expressed as $\overline{\mathrm{X}} \pm$ s. Statistical analysis was performed with Fisher's exact test for $2 \times 2$ tables, and with Pearson's $\chi^{2}$ test for non- $2 \times 2$ tables. Time-to-event curves were estimated by the Kaplan-Meier method and compared using the log-rank test. Cox regression analysis was used to assess the prognostic value of Basigin. $\mathrm{p}<0.05$ was considered to be statistically significant.

\section{Results}

Semiquantitative RT-PCR analysis of Basigin. Total RNA was extracted from the GBC-SD, control RNAi and Basigin/RNAi cells, and the RT-PCR products were electrophoresed on 1.5\% agarose gels. As shown in Fig. 1A, the PCR product of Basigin was $492 \mathrm{bp}$. B-actin was used as the internal control and is the signal seen at $169 \mathrm{bp}$. The gray scale ratios of Basigin/ B-actin are shown in Fig. 1B. These indicate that the Basigin mRNA level was significantly higher in GBC-SD cells than in normal HGBEC cells ( $p<0.001$ ). Moreover, pSUPER/Basigin siRNA transfected into GBC-SD cells markedly reduced Basigin gene expression levels compared with the untransfected or control RNAi transfected cells (both $\mathrm{p}<0.001$ ).

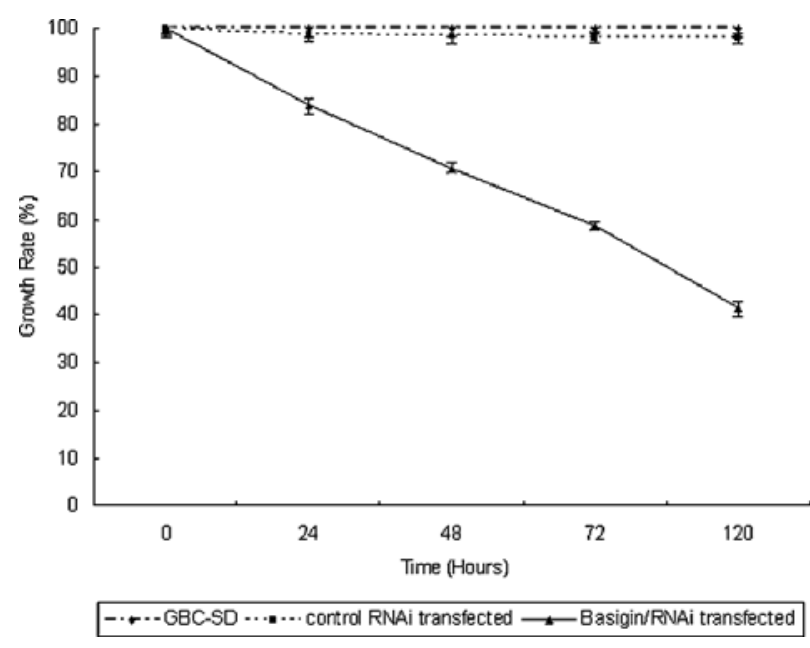

Figure 2. Silencing of Basigin impedes GBC-SD cell proliferation in vitro. Cells were harvested at 24, 48, 72 and $120 \mathrm{~h}$ post-transfection, and cell proliferation was measured by MTT assay. Decreased growth ability was detected in Basigin/RNAi transfected cells compared with untransfected GBC-SD and RNAi control transcfected cells.

Silencing of Basigin inhibits GBC-SD cell proliferation. Because of the importance of the proliferative ability of tumor cells in tumor progression, the in vitro proliferative ability of Basigin-deficient cells was determined by MTT assay. The results indicate that the silencing of Basigin suppressed tumor cell growth. As shown in Fig. 2, Basigin/RNAi cells experienced an $\sim 50 \%$ inhibition rate at the $72 \mathrm{~h}$ time point. Meanwhile, the growth of GBC-SD cells transfected with RNAi control was not affected. Thus, silencing Basigin could inhibit the proliferative ability of GBC-SD in vitro.

Silencing of Basigin inhibits GBC-SD cell invasion and migration. To determine the possible role of Basigin in tumor progression, the capacity of GBC-SD cells for invasion or migration after RNAi treatment was assessed using a modified Bowden chamber assay with a Fluoroblok membrane. Only cells that migrated or invaded across the membrane were fully recorded, in order to quantify the invasive and metastatic capacity of the cells in real time. As shown in Fig. 3, there was a sharp increase in fluorescence during the first $12 \mathrm{~h}$ for the GBC-SD, control RNAi transfected and Basigin/RNAi transfected cells. From 12 to 48 h, the fluorescence generated by the Basigin/RNAi transfected cells decreased significantly, whereas that of the GBC-SD and control RNAi transfected cells increased $(\mathrm{p}<0.01)$. For the invasion assay, the fluoroblok membrane was coated with Matrigel. As with the migration assay, the fluorescence increased sharply during the first $12 \mathrm{~h}$, then leveled off in the case of the Basigin/RNAi transfected cells, the invasion capacity of which appeared to be dramatically reduced compared to the controls $(\mathrm{p}<0.01)$.

Association between Basigin expression and the clinicopathological characteristics of GC. Basigin was mainly detected in cancerous tissues, but was also expressed in some chronic cholecystitis patients. Of the 98 patients, 80 (81.63\%) with GC exhibited Basigin-positive expression, whereas only 2 of 26 patients $(7.69 \%)$ with chronic cholecystitis did. 

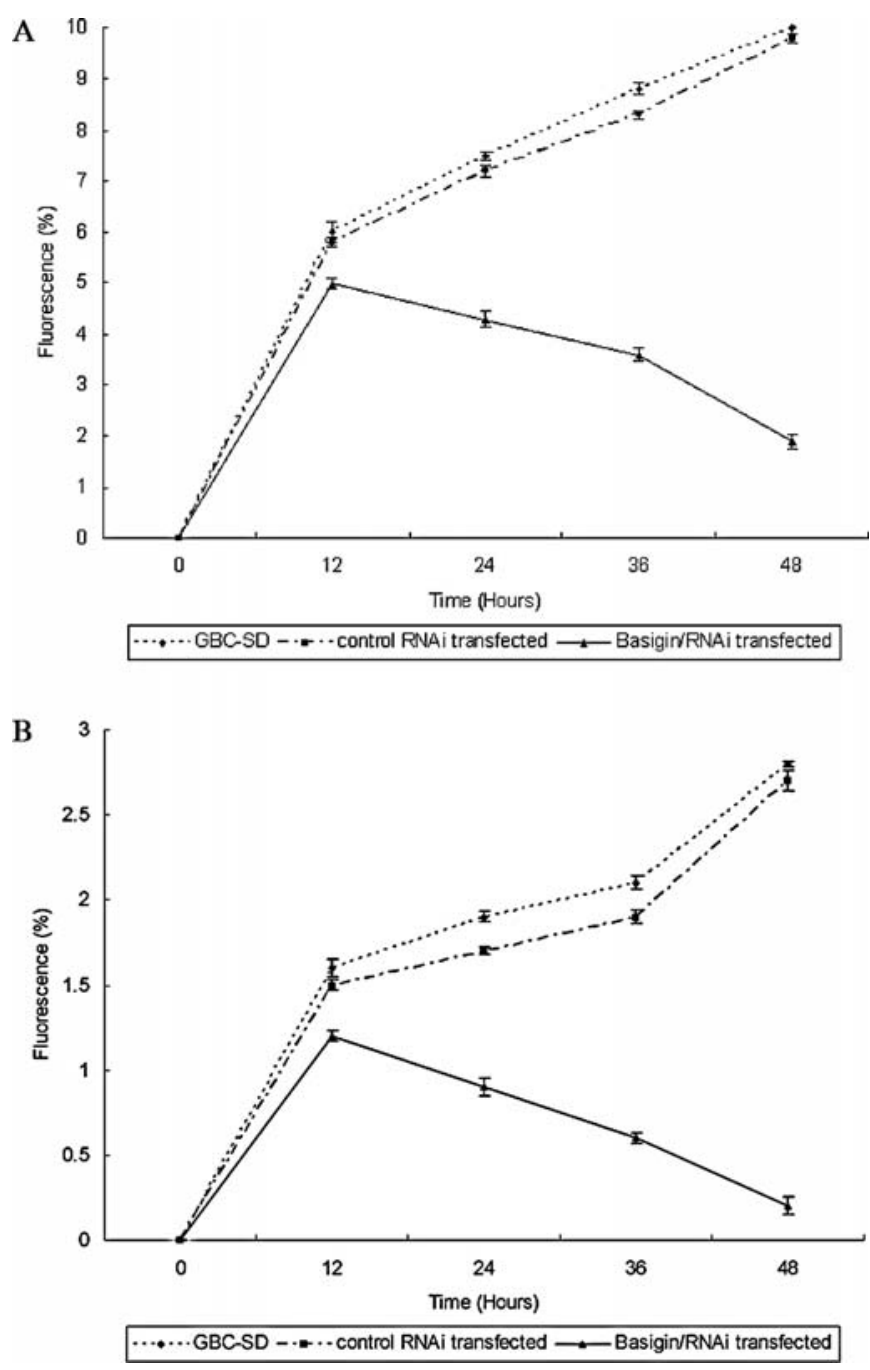

Figure 3. Silencing of Basigin inhibits GBC-SD cell (A) migration or (B) invasion. Each experiment was repeated three times, and each point represents the mean $\pm \mathrm{SD}$ of three separate determinations.
The association between Basigin expression and the clinicopathological features of the GC patients is shown in Table I. The positive expression of Basigin was not related to the gender or tumor size of the GC patients. Positive Basigin expression tended to be positively associated with the Nevin stage of the tumor tissue $(\mathrm{p}<0.01)$, as well as with the degree of histological differentiation ( $\mathrm{p}=0.02$ ). In particular, the incidence of Basigin-positive expression was significantly higher in tumor tissues with distant metastasis than in those without it $(\mathrm{p}<0.01)$.

The prognostic role of Basigin expression patterns in patients with gallbladder carcinoma. The 98 patients were subject to a 5-year follow-up. In terms of the Nevin stages of GC, the 5-year survival rate of patients with Basigin expression was lower than that of those without its expression $(\mathrm{p}<0.01$, Table II). Moreover, in the carcinomas with distant metastasis, patients with positive Basigin expression had a significantly lower 5-year survival rate than those with negative expression $(\mathrm{p}<0.01$, Table II). However, there was no significant association between Basigin expression and the 5-year survival rate of patients with all three degrees of histological differentiation $(\mathrm{p}>0.05)$.

The association between the 5-year survival rates and the expression levels of Basigin was analyzed using the KaplanMeier method. According to their Basigin expression levels, the patients were classified into three groups: 0,1 and 2 . The $\chi^{2}$ value by the log-rank test (Mantel-Cox) indicated a significant difference among the groups with regard to the expression status of Basigin ( $\mathrm{p}<0.01$, Table III). In all three groups, patients with stronger Basigin-positive expression had the poorest prognosis. Cox regression analysis revealed the expression of Basigin to be an apparent independent prognostic indicator (Fig. 4).

Table I. Association between Basigin expression and the clinicopathological features of the gallbladder carcinoma patients.

\begin{tabular}{|c|c|c|c|c|c|}
\hline \multirow[b]{2}{*}{ Factor } & \multirow[b]{2}{*}{ No. } & \multicolumn{3}{|c|}{ Basigin expression $(\%)$} & \multirow[b]{2}{*}{ p-value } \\
\hline & & 0 & 1 & 2 & \\
\hline \multicolumn{6}{|l|}{ Gender } \\
\hline Male & 58 & 17.24 & 44.83 & 37.93 & \multirow[t]{2}{*}{0.16} \\
\hline Female & 40 & 20.00 & 50.00 & 30.00 & \\
\hline \multicolumn{6}{|c|}{ Tumor size $(\mathrm{cm})$} \\
\hline$\geq 5.0$ & 55 & 18.18 & 51.86 & 30.96 & \multirow[t]{2}{*}{0.38} \\
\hline$<5.0$ & 43 & 18.60 & 50.91 & 30.91 & \\
\hline \multicolumn{6}{|l|}{ Nevin stages } \\
\hline I-III & 57 & 21.05 & 60.88 & 18.07 & \multirow[t]{2}{*}{$<0.01$} \\
\hline IV-V & 41 & 14.63 & 41.46 & 43.90 & \\
\hline \multicolumn{6}{|c|}{ Distant metastasis } \\
\hline M0 & 56 & 21.43 & 60.21 & 20.14 & \multirow[t]{2}{*}{$<0.01$} \\
\hline M1 & 42 & 14.29 & 45.24 & 38.10 & \\
\hline \multicolumn{6}{|c|}{ Histological type } \\
\hline Well & 39 & 23.08 & 51.28 & 25.64 & \multirow[t]{3}{*}{0.02} \\
\hline Moderate & 31 & 22.58 & 54.84 & 22.58 & \\
\hline Poor & 28 & 7.14 & 32.14 & 60.71 & \\
\hline
\end{tabular}


Table II. Kaplan-Meier analysis of the postoperative survival rate according to Basigin expression in the cancer tissues.

\begin{tabular}{lrrrr}
\hline & \multicolumn{3}{c}{ 5-year survival rate (\%) } \\
$\begin{array}{l}\text { Clinicopatho- } \\
\text { logical features }\end{array}$ & No. & Basigin & Basigin $^{+}$ & p-value \\
\hline Nevin stage & & & & \\
$\quad$ I-II & 57 & 81.26 & 42.18 & 0.02 \\
IV-V & 41 & 50.23 & 10.86 & $<0.01$ \\
Distant metastasis & & & & \\
M0 & 56 & 82.08 & 41.02 & 0.03 \\
M1 & 42 & 36.11 & 9.26 & $<0.01$ \\
\hline
\end{tabular}

Table III. Kaplan-Meier analysis of the prognostic value of Basigin expression.

\begin{tabular}{lccc}
\hline \multirow{2}{*}{ Group } & Total no. & \multicolumn{2}{c}{ 5-year survival rate } \\
\cline { 3 - 4 } & 18 & 15 & No. \\
\hline 0 & 46 & 25 & 83.33 \\
1 & 34 & 10 & 54.35 \\
2 & 98 & 50 & 29.41 \\
Overall & & & 51.02 \\
\hline
\end{tabular}

\section{Discussion}

Basigin has tumor-enhancing potential in gallbladder carcinomas. The specific knock-down of target genes without affecting other genes is critically important in research on gene function. RNAi is a specific gene-silencing technology (11). In the present study, we constructed the Basigin siRNA expression vector $\mathrm{pSUPER} /$ Basigin siRNA for transfection into the malignant gallbladder carcinoma (GC) cell line GBC$\mathrm{SD}$, which can be effectively and stably inhibited to express the Basigin gene.

The presence of malignant invasion and metastasis, either at diagnosis or later in the clinical course, is one of the most frequent clinical findings in GC. The growth and invasion of $\mathrm{GC}$ cells involves the breakdown of the extracellular matrix by proteinases including matrix metalloproteinases (MMPs), which are involved in tumor progression, growth, invasion, angiogenesis and metastasis $(12,13)$. Previous studies have demonstrated that Basigin promotes tumor cell invasion by stimulating stromal cells to produce elevated levels of MMPs (14-16). As shown in the present study, interference with Basigin expression suppressed the proliferative potential of GC cells in vitro. In agreement with a recently published study $(17,18)$, we found up-regulated expression of Basigin mRNA in GC cells, suggesting its participation in the process of proliferation as an essential component of cell surface signaling transduction. Li et al (19) and $\mathrm{Xu}$ and Hemler (20) showed a strong association between cell proliferation and the BasiginCD98 cell surface super complex, which plays a critical role in energy metabolism. Furthermore, we demonstrated that

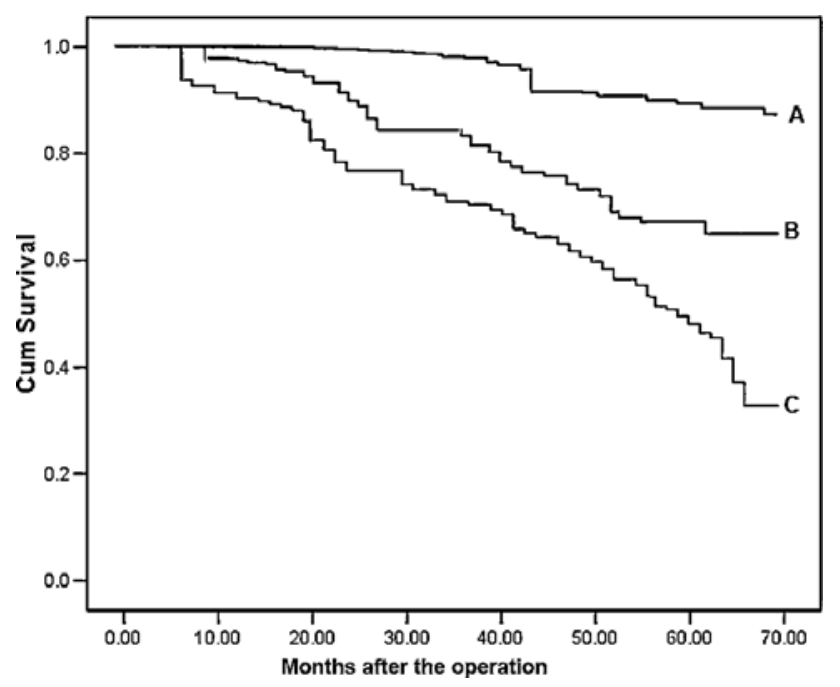

Figure 4. Kaplan-Meier survival curves for Basigin expression in GC. (A) Categorized by negative Basigin expression (18 cases); (B) categorized by weak-moderate positive Basigin expression (46 cases); (C) categorized by stronger positive Basigin expression (34 cases). Survival was significantly poorer for patients with stronger positive Basigin expression than for the other two groups $(\mathrm{p}<0.01)$.

Basigin siRNA inhibits the invasiveness as well as the metastasic capacity of GC cells in vitro. Our findings indicate that Basigin is involved in the invasion and metastasis of GC cells.

Basigin has a poor prognostic role in gallbladder carcinomas. In the present study, we determined the expression of Basigin in cancerous tissue by immunohistochemical staining, and explored the association between this expression, clinicopathological findings and the 5-year survival rate in 98 patients with GC. GC tissues had a heterogeneous staining pattern of weakly-, moderately- and strongly-expressed Basigin, while chronic cholecystitis tissues did not. These abnormal categories did not show a statistically significant association with several commonly assayed factors, including tumor size, age and patient gender, but the association with histological type, Native stage and lymphatic metastasis status was significant.

In this study, the prognosis for Basigin-positive patients with GC was found to be significantly worse than the prognosis for Basigin-negative ones. The results are consistent with a previous study on other types of carcinoma. The positive association of Basigin expression with Nevin stage, distant metastasis and the degree of histological differentiation found in this study may explain the poor prognostic outcome conferred by Basigin expression.

Multivariate analysis of Basigin revealed that the classification of Basigin expression had independent prognostic value when the other factors considered, including Nevin stage, distant metastasis and the degree of histological differentiation, were taken into account. In the present study, we analyzed the association of Basigin expression with the prognosis of GC. Significant differences in prognosis were found among the three groups with different Basigin expression. Patients without Basigin expression had the best outcome, while those with strong Basigin expression had the worst.

In conclusion, our findings indicate that Basigin-targeting siRNA could significantly downregulate the Basigin mRNA 
level in GC cells, and that it inhibits their proliferation, invasiveness and metastatic activity in vitro. The suitability of Basigin as a prognostic marker and therapeutic target for earlystage GC was also evaluated.

\section{Acknowledgements}

This work was supported by grants from the Natural Science Foundation of Hunan Province of China (No. 04JJ3100), the National High Technology Research and Development Program of China (No. 2001BA703BO), and the Hunan Science and Technology Committee (No. 05SK3052).

\section{References}

1. Jemal A, Murray T, Ward E, et al: Cancer statistics. CA Cancer J Clin 55: 10-30, 2005.

2. Ito H, Matros E, Brooks DC, et al: Treatment outcomes associated with surgery for gallbladder cancer: a 20 -year experience. J Gastrointest Surg 8: 183-190, 2004.

3. Cubertafond P, Gainant A and Cucchiaro G: Surgical treatment of 724 carcinomas of the gallbladder. Results of the French Surgical Association Survey. Ann Surg 219: 275-280, 1994.

4. Chang HJ, Yoo BC, Kim SW, et al: Significance of PML and p53 Protein as molecular prognostic markers of gallbladder carcinomas. Pathol Oncol Res 13: 326-335, 2007.

5. Misra S, Chaturvedi A, Goel MM, et al: Overexpression of p53 protein in gallbladder carcinoma in North India. Eur J Surg Oncol 26: 164-167, 2000.

6. Pulukuri S, Patel J, Estes N, et al: Matrix metalloproteinase-1 (MMP-1) expression and biological significance in the progression of prostate cancer. American Association for Cancer Research Annual Meeting: pp14-18, 2007.

7. Nabeshima K, Suzumiya J, Nagano M, et al: Emmprin, a cell surface inducer of matrix metalloproteinases, is expressed in T-cell lymphomas. J Pathol 202: 341-351, 2004.

8. Sidhu SS, Mengistab AT, Tauscher AN, et al: The microvesicle as a vehicle for EMMPRIN in tumorstromal interactions. Oncogene 23: 956-963, 2004.
9. Millimaggi D, Mari M, D'Ascenzo S, et al: Tumor vesicleassociated CD147 modulates the angiogenic capability of endothelial cells. Neoplasia 9: 349-357, 2007.

10. Tang Y, Marian T, Nakada MT, et al: Extracellular matrix metalloproteinase inducer stimulates tumor angiogenesis by elevating ascular endothelial cell growth factor and matrix metalloproteinases. Cancer Res 65: 3193-3199, 2005.

11. Chen X, Lin J, Kanekura T, et al: A small interfering CD147targeting RNA inhibited the proliferation, invasiveness and metastatic activity of malignant melanoma. Cancer Res 66: 11323-11330, 2006.

12. Davidson B, Goldberg I and Berner A: EMMPRIN (extracellular matrix metalloproteinase inducer) is a novel marker of poor outcome in serous ovarian carcinoma. Clin Exp Metastasis 20: $161-169,2003$

13. Tsai WC, Chao YC, Sheu LF, et al: EMMPRIN and fascin overexpression associated with clinicopathologic parameters of pancreatobiliary adenocarcinoma in Chinese people. APMIS 115: 929-938, 2007.

14. Sun A and Hemler M: Regulation of MMP-1 and MMP-2 production through CD147/Extracellular Matrix Metalloproteinase Inducer Interactions. Cancer Res 61: 2276-2281, 2001.

15. Zucker S, Hymowitz M, Rollo E, et al: Tumorigenic potential of extracellular matrix metalloproteinase inducer. Am J Pathol 158: 1921-1928, 2001

16. Tang Y, Kesavan $\mathrm{P}$ and Marian T: Tumor-Stroma interaction: positive feedback regulation of extracellular matrix metalloproteinase inducer (EMMPRIN) expression and matrix metalloproteinase-dependent generation of soluble EMMPRIN. Mol Cancer Res 2: 73-80, 2004.

17. Mi Z, Oliver T, Guo H, et al: Thrombin-cleaved $\mathrm{COOH}(-)$ terminal osteopontin peptide binds with cyclophilin C to CD147 in murine breast cancer cells. Cancer Res 67: 4088-4097, 2007.

18. Riethdorf S, Reimers N, Assmann V, et al: High incidence of EMMPRIN expression in human tumors. Int J Cancer 119: 1800-1810, 2006

19. Li M, Zhai Q, Bharadwaj U, et al: Cyclophilin A is overexpressed in human pancreatic cancer cells and stimulates cell proliferation through CD147. Cancer 106: 2284-2294, 2006.

20. Xu D and Hemler ME: Metabolicac tivation-related CD147-98 complex. Mol Cell Proteomics 4: 1061-1071, 2005. 